Running Head: Right PFC and Luria’s Fist-Edge-Palm task

\title{
1 What type of inhibition underpins performance on Luria's Fist-Edge-Palm task?
}

2 Varkovetski, Michael ${ }^{1}$., Pihkanen, Kaisla ${ }^{1}$., Shanker, Shanti ${ }^{1,2}$., Parris, Benjamin. $\mathrm{A}^{1^{*}}$., \& Gurr, 3 Birgit $^{2}$.

4 1. Department of Psychology, Bournemouth University

5 2. Community Brain Injury and Adult Neuropsychology Poole Hospital, Dorset Healthcare

6 University NHS Foundation Trust

7

8 *Address for correspondence:

9 Dr. Benjamin A. Parris

10 Department of Psychology

11 Faculty of Science and Technology

12 Bournemouth University

13 Talbot Campus

14 Fern Barrow

$15 \quad \mathrm{BH} 12$ 5BB

16 Email: bparris@bournemouth.ac.uk 


\section{Abstract}

Objective: The Fist-Edge-Palm task is a motor sequencing task believed to be sensitive to frontal lobe impairment. The present study aimed to investigate the inhibitory processes underlying successful execution of this task.

Method: Seventy-two healthy participants were asked to perform the Fist-EdgePalm task paced at 120bpms, 60bpms and self-paced. They also completed assessments sensitive to recently dissociated forms of inhibition (the Hayling respectively), and Cattell's Culture Fair Intelligence test.

Results: Analysis revealed that performance on the Hayling Sentence Completion Test predicted the amount of crude errors and the overall score on the Fist-Edgeperformance on the Fist-Edge-Palm task.

Conclusions: Consistent with some previous neuroimaging findings, the present findings suggest that Fist-Edge-Palm task performance relies on right lateralised inhibitory processes. 

sequencing 


\section{Public Significance Statement:}

43 Luria's Fist-Edge-Palm task is a well-known neuropsychological assessment employed to assess

44 frontal lobe and psycho-motor functioning, and to detect voluntary movement disorders, but the

45 inhibitory processes underpinning performance are not well understood. This study provides

46 evidence indicating that right, but not left, prefrontal cortex inhibition functions underpin

47 successful performance on Luria's task. These findings increase the clinical utility of this much48 used task. 


\section{Introduction}

Human voluntary movement is the outcome of a highly complex functional system which

53 incorporates a multitude of cognitive processes relying on the synchronous organization and

54 utilization of various cortical regions (Miziara, Manreza, Mansur, Reed \& Buchpiguel, 2013),

55 and as such a variety of neuropsychological assessments are critical to making fine distinctions

56 of an individual's cognitive and motor abilities. One well-known and widely used task is the

57 Fist-Edge-Palm task (FEP; Luria, 1966). The FEP task is a complex motor sequence task

58 developed to assess frontal lobe and psycho-motor functioning and has been extensively utilized

59 to detect voluntary movement disorders (Umetsu et al., 2002) and is included in numerous

60 neuropsychological assessment batteries (Chen et al., 1995; Dubois, Slachevsky, Litvan, \&

61 Pillon, 2000; Golden, 1981; Mitsuhashi, Hirata, \& Okuzumi, 2018). The task relies on fine motor

62 coordination and a number of executive functions such as planning, updating and inhibition

63 (Chan et al., 2015). During the FEP task, participants are required to reproduce a sequence of

64 hand movements presented by the examiner, most commonly in the "fist-edge-palm"

65 arrangement. Participants are then asked to repeat the sequence of hand movements for a certain

66 number of cycles. A single cycle is comprised of a fist with the knuckles down, followed by a

67 cutting motion with the fingers fully extended, and concludes with a flat palm on the table with

68 the fingers fully extended. Participants are required to break contact with the table between each

69 change in hand movement.

Whilst there has been much work investigating the neural correlates of the FEP task

71 (Astolfi et al., 2004; Chan, Rao, Chen, Ye \& Zhang, 2006; Chan et al., 2015; Rao et al., 2008;

72 Serrien \& Brown, 2003; Umetsu et al., 2002) there is a surprising dearth of research on the

73 cognitive mechanisms underpinning the FEP task. A central challenge inherent in correct 
74 performance of the FEP task is the inhibition of the prepotent but incorrect hand movements

75 associated with the task. Participants must not perform the flat palm movement after the fist-

76 with-knuckles-down movement. Yet it is clear that there are varying levels of success at

77 implementing this form of inhibitory control (Weiner et al., 2011). Kok (1999) reviewed

78 behavioural and psychophysiological studies and concluded that there are multiple forms of

79 inhibition with distinct and interacting neuronal substrates. For example, Van veen and Carter

80 (2005), and more recently Parris et al. (2019), have argued for separate neural substrates for two

81 distinct types of inhibition in the Stroop Colour-Word Test (Stroop test). Consistently, Cipolloti

82 et al. (2016) have recently proposed that there are several processes controlled by anatomically

83 separable systems involved in inhibition tasks.

$84 \quad$ Cipolotti et al. (2016) systematically explored the relationship between inhibition, fluid

85 intelligence and lesion location in a neuroimaging study employing voxel-based lesion-symptom

86 mapping. The results from 30 frontal lobe patients of varying aetiologies showed that after

87 accounting for fluid intelligence (as measured by Cattel's Culture Fair Intelligence test),

88 performance on the Hayling Sentence Completion test (Hayling test; Burgess \& Shallice, 1996),

89 which requires participants to finish a sentence with a word that is not related to the sentence's

90 meaning (e.g., The captain wanted to stay with the sinking....lamp) significantly relied on the

91 integrity of the right Prefrontal Cortex (PFC), specifically the superior and middle frontal gyri. In

92 contrast, performance on the Stroop test (Stroop, 1935), which requires participants to name the

93 colour of the ink in which a word is presented (e.g., the word red is written in blue ink) relied on

94 the integrity of the superior and middle frontal gyri of the left PFC. The authors noted that these

95 findings are consistent with other findings in the literature (Aron, Robbins, \& Poldrack, 2014;

96 Demakis, 2004; Derrfuss, Brass, Neumann, \& von Cramon, 2005; Geddes et al., 2014; Hodgson 
97 et al., 2007; Hornberger, Geng, \& Hodges, 2011; Parris et al., 2019; Perret, 1974; Robbins, 2007;

98 Robinson et al., 2015; Roca et al., 2010; Stuss et al., 2001; Szczepanski \& Knight, 2014) and

99 argued that lesion location is critical in producing impairments on two inhibitory tasks that

100 despite loading similarly on verbal control, have different neurological substrates. Moreover, the

101 authors argued that the two measures of inhibition are therefore possibly dissociable components

102 of the executive function of inhibition, supporting Kok's (1999) conclusion that there are

103 multiple forms of inhibition.

104 The aim of the present study was to investigate whether the distinct inhibitory

105 mechanisms involved in the Hayling and Stroop tasks underpin performance on the FEP task.

106 Given their uniquely and recently established doubly dissociated inhibitory mechanisms

107 (Cipolotti et al., 2016), we investigated whether one or both cognitive tasks predicted FEP task

108 performance. Whilst both the Hayling and Stroop tests are measures of lexical control, their

109 established dissociation suggests important differences between the two tasks (see the Discussion

110 section for a fuller consideration of this issue) and any association with FEP task performance

111 would be informative as to the cognitive mechanisms underpinning this commonly used motor

112 sequencing task. Following Cipolotti and colleagues (2016), a measure of fluid intelligence was

113 included in our analysis as a measure of general cognitive ability. Fluid intelligence was included

114 in our analysis because it has been shown to partially mediate performance on the Hayling test

115 (Martin, Barker, Gibson, \& Robinson, 2013) and could thus potentially be responsible for any

116 relationships between Hayling and FEP performance.

117 Evidence for a right PFC locus for FEP performance in neuroimaging work (Rao et al.,

118 2008) suggests that FEP performance might rely on similar inhibitory control mechanisms as

119 those underpinning the Hayling test. To investigate this potential relationship and to sufficiently 
120 tax the capacities of our healthy participants we titrated task difficulty by asking participants to

121 perform the FEP task in three pacing conditions. It was reasoned that the self-paced condition

122 would lead to ceiling effects and so we introduced two externally paced conditions; one paced at

$12360 \mathrm{bpm}$ and one at $120 \mathrm{bpm}$. It was expected that the externally-paced conditions would be harder

124 than the self-paced condition, and of the externally-paced conditions, the faster condition

$125(120 \mathrm{bpm})$ would be harder than the slower condition $(60 \mathrm{bpm})$; it was reasoned that we might be

126 more likely to observe a relationship between FEP task performance and the Hayling and / or

127 Stroop tests in a healthy population if the task was more difficult. However, since this was not a

128 key prediction in our investigation (indeed we were unsure as to how or whether pacing a

129 condition would modify performance in healthy controls) it was a priori decided only to analyse

130 the pacing conditions as separate conditions if an initial one-way ANOVA or non-parametric

131 equivalent and appropriate follow up tests assessing differences between performance for the

132 three levels of pacing returned a significant result. This constraint would have the effect of

133 reducing the need for multiple analyses for each performance measure (subtle errors, crude

134 errors, and self-corrections of those errors, and an overall FEP score).

\section{2. Methods}

\subsection{Design}

This study utilized a repeated measures design. Scores on the Stroop, Hayling and

138 Cattell's Culture Fair Intelligence tests were the independent variables. The amount of subtle

139 errors, crude errors, self-corrections, and the overall score on the FEP task were the dependent

140 variables. 


\subsection{Participants}

Seventy-two healthy university students (45 females and 27 males; mean age $=21$ years,

$143 \mathrm{SD}=3.30-$ see Table 1) were recruited from the Psychology Research Participation System at

144 Bournemouth University. All participants reported no neurological illness or psychiatric

145 diseases. The Bournemouth University ethics panel approved this study. Participants received an

146 information sheet prior to consenting and were debriefed at the end of the study. Written

147 informed consent was obtained for every participant.

\subsection{Materials}

To measure left PFC performance, we used the Golden and Freshwater (2002) version of

150 the Stroop Colour-Word Test which assesses prepotent response inhibition. The test consisted of

151 three sections; each section arranged into five columns which consisted of 20 items each. The

152 first section consisted of 100 words in black ink, the second section of 100 lines of "XXXX"

153 printed in coloured ink (blue, red and green), and the third section of 100 words "BLUE",

154 "RED" and "GREEN" printed in an incongruent colour. In the first section, participants were

155 instructed to read the words out loud as quickly as possible. In the second section, participants

156 were instructed to name the colour of the ink for each item as quickly as possible. In the third

157 section, participants were instructed to say out loud the ink colour of each word. Participants

158 were instructed to complete each section as quickly as possible within a time limit of 45 seconds.

159 If participants reached the end of the last column before the time limit, they were instructed to

160 reread the page. Participants were not permitted to cover the page by any means, or to use their

161 finger to guide their gaze. Whilst we employed the Golden and Freshwater (2002) version of the

162 Stroop task (this was the version available to us) and used their recommended time limit,

163 following Cipolotti and colleagues (2016) we calculated a single score based on the amount of 
164 correctly identified incongruent ink colours in the third section, within this time limit (Trenerry,

165 Crosson, DeBoe and Leber, 1989).

166 The Hayling Sentence Completion Test (Burgess \& Shallice, 1997), which assesses

167 initiation speed and response suppression, is comprised of two sections. In the first section,

168 participants orally completed 15 sentences missing the last word by generating a word which

169 correctly completes each sentence. In the second section, participants orally completed another

17015 uncompleted sentences, but were instructed to generate a word that was unconnected to the

171 sentence in every way. Responses and response time were noted for each sentence. Following

172 Cipolotti and colleagues (2016), we calculated two scores for the second section: the total

173 Suppression Reaction Time and the Suppression Errors Score. The Suppression Errors score is

174 the sum of the Total Category A Errors (errors which plausibly complete the sentence were given

175 a score of 3) and the Total Category B Errors (errors which were somewhat connected to the

176 sentence were given a score of 1). Whilst these scores can be scaled, doing so in a non-patient

177 population leads to very little variability and as such we used the raw scores for all analyses.

178 The Cattell's Culture Fair Intelligence Test (Advanced version, Scale 3, Cattell, 1963)

179 was used as a measure of fluid intelligence. The test is comprised of four subtests: classification,

180 series, matrices and analogies. Each subtest was timed: three minutes for the first, four minutes

181 for the second, three minutes for the third, and two and a half minutes for the fourth.

182 Participants' correct answers were summed up for each subtest to give a final score which was

183 then scaled to give an estimate of fluid intelligence.

\subsection{Procedure}

185 The FEP task lacks a standardized administration protocol and scoring scheme. Luria 186 (1980) provided three administrative steps: first the examiner demonstrates for 10 cycles, then 
187 the patient imitates the examiner for 20 cycles, and finally the patient continues without model 188 for 20 cycles. Despite Luria's initial protocol, variation in administration of the FEP became 189 evident. Several studies have not determined the amount of cycles a participant is required to 190 complete (Rao et al., 2008; Chan et al., 2006), while other clinical studies have asked patients to 191 perform as few as three cycles of the task (Iseki et al., 2013; Weiner et al., 2011), some six (Park 192 \& Moon, 2014; Miziara et al., 2013) and others 15 (Zaytseva et al., 2014). Given this 193 inconsistency we selected a rough mid-way point between previous studies and elected to have 194 our participants perform 10 cycles in each of the pacing conditions.

195 Participants were assessed on the FEP task using either the left or right hand. The first 196 half of the sample was administered the FEP task using the right hand, and the second half of the 197 sample was administered the FEP task using the left hand. Performance was counterbalanced in 198 this way because each hand is controlled by the primary motor cortex in the contralateral 199 hemisphere of the brain and so the relationship to tasks primarily recruiting the left (Stroop) or 200 right (Hayling) hemisphere could otherwise potentially confound the outcome. Prior to 201 administrating the FEP task, a simple pre-test was performed. Participants performed each of the 202 individual motor movements within the FEP to demonstrate that no primary motor deficits were 203 present.

204 Participants were first requested to observe and then imitate the examiner producing a 205 single FEP cycle (fist-edge-palm). Participants were then asked if they understood how to 206 perform the task correctly. Following this, the participants were asked to produce one FEP cycle 207 on their own. Participants were then asked to perform 10 FEP cycles at three different tempos; at 208 their own tempo, an externally paced tempo of 60 beats per minute, and an externally paced 209 tempo of 120 beats per minute. The examiner made a video recording of the hand performing the 
210 FEP task throughout all three tempos. Instructions regarding what to do in case of an error were

211 explained to the participant prior to the start of the experiment. If a participant made a subtle

212 error in technique or from hesitation/lag, they were instructed to continue through their current

213 cycle and to begin the next cycle normally. However, if the participant made a crude error in

214 producing the wrong hand movement, they were instructed to stop and restart that cycle, and to

215 continue onto the next cycle normally. Additionally, participants were asked not to externally

216 narrate they own execution of the FEP task by saying "fist-edge-palm". The examiner kept count

217 of the number of completed cycles for each tempo and asked the participant to stop when they

218 completed 10 cycles. The order of tempos and which hand the participant used was

219 counterbalanced to reduce any order and handedness effects. Due to counterbalancing, and a

220 lack of an equal number of left hand dominant vs. right hand dominant participants, hand

221 dominance was not accounted for in the analysis.

222 Following the completion of all three tempos of the FEP task, the participants' cognitive

223 performance was assessed using the Stroop, Hayling and Culture Fair Intelligence tests. All three

224 tests were administered in the published standard manner and administration was

225 counterbalanced across participants.

226

227

228

229

230

231

\subsection{FEP task scoring}

Variation exists in how the scores were calculated in previous studies. Numerous studies scored only crude errors, such as omission or repetition of a motion (Park \& Moon, 2014;

Miziara et al., 2013). Other studies scored more subtle technical errors, such as flexing of the fingers during cutting motions (Weiner et al., 2011). Furthermore, some studies implemented a point system when scoring errors. In this system, the score is dependent on how many 
232 crude/subtle errors are made (Zaytseva et al., 2014), or how many successful consecutive cycles

233 the patient completes (Iseki et al., 2013).

234 For this reason, in the present study, we created a new method for scoring performance.

235 Subtle errors, crude errors, and self-corrections of those errors and an overall FEP score were

236 used as the dependent variables and were calculated upon reviewing each participant's video

237 recording. The amount of subtle and crude errors a participant made was scored by two

238 researchers. If a disagreement arose on the scoring of any of the indices of performance, they

239 would re-watch the video until an agreement was reached. A subtle error was scored when a

240 participant produced a hand movement with poor technique, or when a hesitation/lag was evident

241 between hand movements. Poor technique is defined as a hand movement with; a fist orientated

242 the wrong way, an edge with the fingers curled in, or a palm that is angled more than $45^{\circ}$ above

243 the table. A crude error was scored when a participant produced the wrong hand motion (e.g., the

244 participant produces a fist instead of a flat palm, following the production of an edge). The

245 amount of self-corrections was also scored. Each subtle error was counted as one point, and each

246 crude error, which we deemed as being a bigger and more problematic error, was counted as two

247 points. Self-corrections were counted as .5 points. To calculate each participant's overall FEP

248 score, the total self-corrections score (across all tempo conditions) was subtracted from the total

249 error score (crude + subtle errors across all tempo conditions).

\section{2.6. Statistical Analysis Plan}

To determine whether either of the four predictors (Hayling test suppression error score,

252 Hayling test suppression reaction score, Stroop test score, or fluid intelligence) were able to

253 significantly predict participants' overall FEP score, we first aimed to conduct a multiple 
254 regression analysis including all measures as predictor variables. We also planned to conduct

255 further multiple regression analyses to determine whether the predictors were able to predict the

256 four dependent variables (crude errors, subtle errors, crude error self-corrections, subtle error

257 self-corrections). However, before conducting the individual analyses of the four dependent

258 variables, we planned to initially determine whether the values of the dependent variables

259 significantly differed between the three tempo conditions (Self-tempo, 60bpm and 120bpm)

260 using a one-way repeated-measures ANOVA and follow up Wilcoxon matched comparisons

261 (with Bonferroni correction). If scores did not significantly differ between the tempo conditions,

262 scores across tempo conditions were combined to reduce the number of analyses conducted. In

263 the event that the DVs were not normally distributed, the non-parametric versions of the tests

264 were used. Finally, in order to establish whether DVs were statistically independent, we planned

265 to run a Kendall rank correlation.

\section{3. Results}

See Table 1 for descriptive data about participants, and Table 2 regarding descriptive data

268 of the neuropsychological assessment scores. See Table 3 for descriptive data regarding FEP task 269 measures.

270 Casewise diagnostics and a scatterplot revealed that one participant was an outlier with

271 an overall FEP score of 33.5 (compared to an average of 8.94). They were removed from the

272 analysis since it was noted during testing that they exhibited difficulties in following the rhythm

273 of the metronome, which may have increased errors, and thus we believe that they were not an

274 accurate representation of the target population. 


\subsection{Analysis of overall FEP score}

To assess linearity, a scatterplot of participants' overall FEP score against each of the

277 four predictor variables with a superimposed regression line was plotted. Visual inspection of

278 these plots revealed a linear relationship between the overall FEP score, and each of the predictor

279 variables. There was homoscedasticity, normality of the residuals and all variance inflation

280 factors were below 1.27 indicating small to nil multicollinearity. With a perfect score of zero, the

281 overall FEP score had a range of scores from zero to 28.5.

282

The four predictor variables accounted for $23 \%$ of the variation in participants' overall

283 FEP score with adjusted $R^{2}=19 \%$, a medium size effect according to Cohen (1988). The four

284 predictor variables significantly predicted the overall FEP score, $F(4,66)=5.03, p=.001$; see

285 Figure 1 . The analysis indicated that only the Hayling test suppression error score significantly

286 predicted the overall FEP score $(\beta=.440, p=.003$; see Figure 2$)$, while Hayling test suppression

287 reaction score $(\beta=.062, p=.253)$, fluid intelligence $(\beta=.066, p=.265)$, and Stroop test score $(\beta$ $288=-.110, p=.198)$ did not.

289

290

291

292

293

294

295

296

297

\subsection{Independent analysis of each dependent variable}

Several of the variables appeared to be relatively rare and significantly skewed. We used P-P plots and indices for acceptable limits of \pm 2 for skewness and kurtosis (Trochim \&

Donnelly, 2006; Field, 2009; Gravetter \& Wallnau, 2014) to check the assumption of normality.

The following variables were shown to be non-normally distributed: Self-Tempo Subtle Errors

$($ Skewness $=2.911$, Kurtosis $=9.977), 60 \mathrm{bpm}$ Subtle Errors $($ Skewness $=1.737$, Kurtosis $=$ 2.397), Self $+60 \mathrm{bpm}$ Subtle Correction (Skewness $=1.706$, Kurtosis $=3.101), 120 \mathrm{bpm}$ Subtle

Corrections $($ Skewness $=2.055$, Kurtosis $=3.942), 60 \mathrm{bpm}$ Crude Errors $($ Skewness $=1.803$, Kurtosis $=2.896), 120 \mathrm{bpm}$ Crude Errors $($ Skewness $=2.938$, Kurtosis $=10.912)$, Self-Tempo 
Crude Corrections $($ Skewness $=2.373$, Kurtosis $=6.214), 60 \mathrm{bpm}$ Crude Corrections $($ Skewness $=$

2.700, Kurtosis = 7.821), 120bpm Crude Correction (Skewness = 2.572, Kurtosis = 7.574),

Overall FEP Score (Skewness = 1.314, Kurtosis = 2.054). Therefore, prior to analysis, we attempted to normalize the variables using log transformations to no success, and thus continued with the non-transformed variables. As a consequence, Friedman's test and Wilcoxon Matched-

303 Pairs tests were employed for analyses of the means.

Furthermore, upon assessing assumptions for regression it was shown that a few variables

305 did not show homoscedastic residuals (self-tempo and 60bpm combined subtle error score,

$306120 \mathrm{bpm}$ subtle error score) and some variables' residuals deviated from normality on the Normal

307 P-P plots (self-tempo and 60bpm combined subtle self-correction score, and 120bpm subtle self-

308 correction score). This could lead to imprecise coefficient estimates and increases the likelihood

309 of a model term that is significant when it is actually not. Therefore, the results from these

310 analyses should be interpreted with caution.

311 Subtle errors: A Friedman test showed that the amount of subtle errors a participant made

312 significantly differed between tempo conditions; $\chi^{2}(2)=37.862, p<.001$. Wilcoxon matched

313 comparisons were performed with a Bonferroni correction for multiple comparisons. Statistical

314 significance was accepted at the $p<.0167$ level. There was a significant difference between the

315 scores for self-tempo subtle errors and 120bpm subtle errors ( $p=.001, r=-0.278)$, and between

$316120 \mathrm{bpm}$ subtle errors and 60bpm subtle errors $(p<.001, r=-0.434)$. There was no significant

317 difference between self-tempo subtle errors and 60bpm subtle errors $(p=.047, r=0.166)$. Thus,

318 two multiple regressions analyses were conducted; the first on a combined score of the self-

319 tempo and $60 \mathrm{bpm}$ subtle errors, and the second on the $120 \mathrm{bpm}$ subtle errors. The results of the

320 multiple regression analysis indicated that neither of the four predictor variables were able to 
321 predict the amount of subtle errors made in the self-tempo and $60 \mathrm{bpm}$ conditions $\left(\mathrm{R}^{2}=.053\right.$,

$322 \mathrm{~F}(4,66)=.918, p=.459)$, or the $120 \mathrm{bpm}$ condition $\left(\mathrm{R}^{2}=.052, \mathrm{~F}(4,66)=.909, p=.464\right)$.

$323 \quad$ Crude errors: A second Friedman test showed that the amount of crude errors a

324 participant made did not significantly differ between each tempo condition; $\chi^{2}(2)=1.589, p=$

325.452 . Thus, a total crude errors score was calculated and used for the multiple regression

326 analysis. The four predictor variables accounted for $24 \%$ of the variation in participants' total

327 crude errors score with adjusted $R^{2}=20 \%$, a medium size effect according to Cohen (1988). The

328 four predictor variables significantly predicted the total crude errors score, $F(4,66)=5.284, p=$

329.001 ; see Figure 3. The analysis indicated that only the Hayling test suppression error score

330 significantly predicted the total crude error score $(\beta=.179, p=.004$; see Figure 4$)$, while

331 Hayling test suppression reaction score $(\beta=.037, p=.107)$, fluid intelligence $(\beta=.029, p=$

$332.249)$, and Stroop test score $(\beta=-.029, p=.424)$ did not.

$333 \quad$ Subtle self-corrections: A third Friedman test showed that the amount of subtle self-

334 corrections a participant made significantly differed between tempo conditions $\chi^{2}(2)=7.189, p=$

335 .027. Pairwise comparisons were once again performed with a Bonferroni correction for multiple

336 comparisons. Statistical significance was accepted at the $p<.0167$ level. A significant difference

337 in the amount of subtle self-corrections a participant made existed between $60 \mathrm{bpm}$ and $120 \mathrm{bpm}$

338 conditions $(p=.002, r=-0.254)$. However, no significant differences in the amount of subtle

339 self-corrections were found between self-tempo and 60bpm conditions $(p=.052, r=0.163)$, or

340 between self-tempo and 120bpm conditions $(p=.318, r=-0.084)$. Thus, two multiple regression

341 analyses were conducted; the first on a combined score of the self-tempo and 60bpm subtle self-

342 correction conditions and the second on the $120 \mathrm{bpm}$ subtle self-correction condition. However,

343 the results of the multiple regression analyses indicated that neither of the four predictors were 
344 able to predict the amount of subtle self-corrections made in the self-tempo and 60bpm

345 conditions $\left(\mathrm{R}^{2}=.035, \mathrm{~F}(4,66)=.600, p=.664\right)$ or the $120 \mathrm{bpm}$ condition $\left(\mathrm{R}^{2}=.044, \mathrm{~F}(4,66)=\right.$

$346 \quad .754, p=.559)$.

347 Crude Self-Corrections: A final Friedman test showed that the amount of crude self-

348 corrections a participant made did not significantly differ between each tempo condition; $\chi^{2}(2)=$

$349.819, p=.664$. Thus, a total crude self-correction score was calculated and used for the multiple

350 regression analysis. Like the analysis of subtle self-corrections, the results of the multiple

351 regression analysis indicated that neither of the four predictors were able to predict the overall

352 amount of crude self-corrections a participant made $\left(\mathrm{R}^{2}=.038, \mathrm{~F}(4,66)=.647, p=.631\right)$.

\section{$353 \quad 3.3$ Correlations between errors}

354 Lastly, a Kendall rank correlation was run to assess the relationship between the subtle 355 and crude errors made during execution of the FEP task. A Kendall rank correlation was chosen

356 due to the violation of the normality assumption among the variables, and because it is

357 considered to be more robust and efficient than the Spearman correlation (Knight, 1996). No

358 significant correlation between total crude errors and subtle errors of each tempo condition was

359 evident. Table 4 summarises these results.

\section{$360 \quad 3.4$ Summary of results}

361 In summary, the analysis indicated that only the Hayling test suppression error score 362 significantly predicted the overall FEP score $(\beta=.440, p=.003$; see Figure 2$)$, while the other

363 IVs did not. Moreover, only the Hayling test suppression error score was able to significantly

364 predict participants total crude error score $(\beta=.179, p=.004$; see Figure 4). 


\section{Discussion}

By assessing performance on the FEP task and neuropsychological assessments sensitive

367 to recently doubly-dissociated inhibitory functions involved in the Hayling and Stroop tests, the

368 present study was able to shed some light on the inhibitory functions underpinning FEP task

369 performance. The Hayling test, a verbal suppression test known for its sensitivity to right PFC

370 lesions (Cipolotti et al., 2016; Robinson et al., 2015), and in particular the suppression score

371 associated with the test, was able to significantly and independently predict FEP task

372 performance, a motor sequencing task, whereas Stroop test performance and fluid intelligence

373 did not significantly predict performance on the FEP task. Other than the overall FEP score, the

374 Hayling test suppression error score was also a significant predictor of crude error scores. There

375 were no other significant predictive relationships between our independent and dependent

376 variables. Overall, our findings provide complimentary cognitive evidence for the involvement

377 of right PFC inhibitory functions in FEP task performance reported in a previous neuroimaging 378 assay (Rao et al., 2008).

Kok (1999) argued that the executive function component of inhibition may comprise

380 multiple forms, each with their own distinct neuronal system. Whilst Cipolotti et al. (2016)

381 argued that their findings supported this assertion by indicating dissociable inhibitory functions,

382 it is not clear how the two types of inhibition measured by these two tasks differ. Cipolotti et al.

383 (2016) described the Stroop test as an inhibitory test that measures the ability to inhibit pre-

384 potent responses, and it could be argued that the Hayling test involves semantic inhibition in that

385 it involves supressing an appropriate semantic response. In fact, whilst the locus of the Stroop

386 effect is commonly attributed to competition between the competing responses that are indicated

387 by each dimension of the Stroop stimulus, it has recently been shown that the Stroop effect 
388 involves competition at various levels of processing including, but not limited to, response,

389 semantic and task level conflict (see Augustinova, Parris \& Ferrand, 2019; Parris, Augustinova

390 \& Ferrand, 2019). Moreover, the type of competition might well depend on whether the Stroop

391 stimuli are presented in blocked or unblocked formats (Hasshim \& Parris, 2017), with the former

392 being more common in the paper version of the task (as used here). For present purposes we

393 interpret the tasks in line with the interpretation of Cipolotti et al. who argued that the Hayling

394 test measures inhibitory mechanisms in the right hemisphere and the Stroop task, inhibitory

395 mechanisms in the left hemisphere. Nevertheless, more research is needed to determine what

396 differentiates the inhibition processes involved in these two tasks.

397 Cipolotti et al. (2016) noted that both tests involve suppressing a dominant response, but

398 also differ in the involvement of other complex processes such as goal maintenance in the face of

399 a visually presented distraction in the case of the Stroop test and strategy utilisation in the

400 Hayling test. Indeed, it could be argued that the FEP task has more in common with the Stroop

401 test in that it requires suppression of a set of manual responses (a set number of possible colour

402 responses in the Stroop test and set number of movements in the Hayling test ). The Hayling test

403 in contrast does not involve inhibition of a manual response and requires the inhibition of just

404 one response. However, in the Stroop test, any of the possible response options could be the next

405 correct response, whereas in the FEP task the next correct response is pre-determined.

406 Maintaining the correct sequence might require the invocation of a strategy such as constantly

407 repeating "fist-edge-palm" to oneself, just as efficient performance on the Hayling test requires

408 strategy use (e.g., use the name of objects in the room as your unrelated response).

409 Unfortunately, our data do not permit a conclusion as to the exact relationship between the 
410 inhibition mechanisms involved in the FEP and Hayling tests, they do however give direction for

411 future research aimed at understanding the mechanisms underpinning the FEP task.

412 A notable limitation of the present research is that our pacing manipulation was not

413 wholly effective. Whilst, as predicted, the $120 \mathrm{bpm}$ condition was shown to be more difficult in

414 terms of errors committed, the self-paced condition was shown to be of equal difficulty to the

$41560 \mathrm{bpm}$ condition. However, the predictive relationship between the Hayling test suppression

416 error score and FEP performance was not dependent on a particular pacing condition.

417 Nevertheless, a future study might consider employing an even faster paced condition to induce

418 more subtle errors and corrections. Such a manipulation might reveal the cognitive processes

419 underpinning more refined errors.

420 Another limitation of the present research is that our method of calculating the Hayling

421 test scores. To score the Hayling test, the number of category A and category B errors are

422 summed and then scaled. Our scaled scores meant that $>90 \%$ of the participants had a score of 6

423 which is clearly not enough variability for our measures to explain. Due to this lack of variability

424 in the Hayling scaled scores, we did not use the scaled scores for either index of Hayling

425 performance. In the interest of maintaining performance variability among participants, we

426 instead used raw scores for all Hayling test analyses. Undoubtedly, this reduces the validity of

427 our analyses. Future studies, particularly those working with clinical populations, might consider 428 using the scaled scores for analysis.

429 A final limitation of the present research is that some participants completed the FEP task

430 with their non-dominant hand. This was the case because it was reasoned that having participants

431 complete the task with only their dominant hand would result in most participants recruiting left

432 hemisphere motor control functions (87.5\% of the participants were right handed), which might 
433 have confounded any relationship with the higher order cognitive control functions involving

434 inhibition whose apparent laterality motivated the present research. Having some participants

435 complete the task with their non-dominant hand might have increased the number of errors in

436 their performance. However, the assumption that the control processes for the nondominant

437 hand are a weaker analogue of those of the dominant arm has been argued to be erroneous and

438 instead research suggests that there are specific advantages for each arm for different aspects of a

439 movement where the left hemisphere specialises in planning and coordinating actions, and the

440 right hemisphere specialises in updating actions and stopping at a goal position (Mutha, Haaland,

$441 \&$ Sainburg, 2012). Nevertheless, future studies might consider recruiting an equal number of left

442 hand dominant and right-hand dominant participants.

$443 \quad$ For the purposes of this research, a new protocol and scoring method for the FEP task

444 was introduced. It is hoped that this method proves useful for future research. However, the

445 protocol and method does present with some shortcomings; meaning it might not be suitable for

446 all future research, particularly research involving patients. First, Luria recommended taking

447 patients through 20 guided cycles of the task before testing their ability to do it independently.

448 We did not do this in the present study precisely because we were using a healthy population.

449 The inhibition mechanisms involved might change after such prolonged practice. Indeed,

450 strategy use might be of less importance and thus could alter the inhibitory mechanisms involved

451 (and the associated neural substrate). Second, whilst the scoring of subtle errors and self-

452 corrections might be informative in a heathy adult population, patient populations would be more

453 likely to make just the crude errors. Notably, however, none of the analyses involving these

454 measures of more refined performance produced significant results, and whilst we must not draw 
455 strong conclusions based on null results, our data do point to the need for predictor variables of 456 an equally refined nature.

457 In summary, our findings suggest that performance on the FEP task can be predicted by 458 performance on the Hayling Sentence Completion test, and that a right PFC inhibitory process is 459 key for the successful execution of the FEP task. Additionally, we believe that the novel and 460 more robust administration protocol and scoring system will be of value to clinicians utilizing the 461 FEP task as a diagnostic tool to measure the magnitude of impairment. Future studies should 462 recruit clinical populations to further develop the FEP scoring system, and to assess its reliability 463 in distinguishing different diagnoses.

464

465

466

467

468

469

470

471

472

473

474

475 
476

477

478

479

480

481

482

483

484

485

486

487

488

489

490

491

492

493

494

495

496

497

498

References

Aron, A. R., Robbins, T. W., \& Poldrack, R. A. (2014). Inhibition and the right inferior frontal cortex: One decade on. Trends in Cognitive Sciences, 18(4), 177-185. doi:10.1016/j.tics.2013.12.003

Astolfi, L., Cincotti, F., Mattia, D., Salinari, S., Babiloni, C., Basilisco, A., . . Babiloni, F. (2004). Estimation of the effective and functional human cortical connectivity with structural equation modeling and directed transfer function applied to high-resolution EEG. Magnetic Resonance Imaging, 22(10), 1457-1470. doi:10.1016/j.mri.2004.10.006

Augustinova, M., Parris, B. A., \& Ferrand, L. (2019). The Loci of Stroop Interference and Facilitation Effects With Manual and Vocal Responses. Frontiers in Psychology, 10. doi: 10.3389/fpsyg.2019.01786

Burgess, P. W., \& Shallice, T. (1996). Response suppression, initiation and strategy use following frontal lobe lesions. Neuropsychologia, 34(4), 263-272. doi:10.1016/00283932(95)00104-2

Burgess, P. W., \& Shallice, T. (1997). The Hayling and Brixton Tests. Bury St Edmunds: Thames Valley Test Company.

Cattell, R. B. (1963). Theory of fluid and crystallized intelligence: A critical experiment. Journal of Educational Psychology, 54(1), 1-22. doi:10.1037/h0046743

Chan, R. C., Rao, H., Chen, E. E., Ye, B., \& Zhang, C. (2006). The neural basis of motor sequencing: An fMRI study of healthy subjects. Neuroscience Letters, 398(3), 189-194. doi:10.1016/j.neulet.2006.01.014

Chan, R. C., Huang, J., Zhao, Q., Wang, Y., Lai, Y., Hong, N., . . Dazzan, P. (2015). Prefrontal cortex connectivity dysfunction in performing the Fist-Edge-Palm task in patients with 
first-episode schizophrenia and non-psychotic first-degree relatives. Neurolmage: Clinical, 9, 411-417. doi:10.1016/j.nicl.2015.09.008

Chen, E. Y., Shapleske, J., Luque, R., Mckenna, P. J., Hodges, J. R., Calloway, S., . . Berrios,

Derrfuss, J., Brass, M., Neumann, J., \& Cramon, D. Y. (2005). Involvement of the inferior

Demakis, G. J. (2004). Frontal Lobe Damage and Tests of Executive Processing: A Meta-

Cohen, J. (1988). Statistical Power Analysis for the Behavioral Sciences (2nd ed.). Hillsdale, NJ:

Cipolotti, L., Spanò, B., Healy, C., Tudor-Sfetea, C., Chan, E., White, M., . . Bozzali, M. (2016). Inhibition processes are dissociable and lateralized in human prefrontal cortex. Neuropsychologia, 93, 1-12. doi:10.1016/j.neuropsychologia.2016.09.018 Lawrence Erlbaum Associates, Publishers. Analysis of the Category Test, Stroop Test, and Trail-Making Test. Journal of Clinical and Experimental Neuropsychology, 26(3), 441-450. doi:10.1080/13803390490510149 frontal junction in cognitive control: Meta-analyses of switching and Stroop studies. Human Brain Mapping, 25(1), 22-34. doi:10.1002/hbm.20127

Dubois, B., Slachevsky, A., Litvan, I., \& Pillon, B. (2000). The FAB: A frontal assessment battery at bedside. Neurology, 55(11), 1621-1626. doi:10.1212/wnl.55.11.1621

Field, A. (2009). Discovering statistics using SPSS. London: SAGE Publications.

Geddes, M. R., Tsuchida, A., Ashley, V., Swick, D., \& Fellows, L. K. (2014). Material-specific interference control is dissociable and lateralized in human prefrontal cortex. Neuropsychologia, 64, 310-319. doi:10.1016/j.neuropsychologia.2014.09.024 
522 Golden, C. J. (1981). The Luria-Nebraska Children's Battery: Theory and formulaiton. In G. W.

523 Hynd \& J. E. Orbrzut (Eds.), Neuropsychological assessment and the school-age child:

$524 \quad$ Issues and procedures (pp. 277-302). New York, NY: Grune \& Stratton.

525 Golden, C. J., \& Freshwater, S. M. (2002). Luria-Nebraska Neuropsychological Battery. In W. I.

526 Dorfman \& M. Hersen (Eds.), Understanding Psychological Assessment (pp. 59-75).

527 Boston, MA: Springer.

528 Gravetter, F. and Wallnau, L. (2014) Essentials of Statistics for the Behavioral Sciences. 8th

$529 \quad$ Edition, Wadsworth, Belmont, CA.

530 Hasshim, N. \& Parris, B. A. (2017). Trial type mixing substantially reduces the response set 531 effect in the Stroop task. Acta Psychologica, 189, 43-53.

532 Hodgson, T. L. Chamberlain, M., Parris, B. A., James, M., Gutowski, N., Husain, M. \& Kennard,

Hornberger, M., Geng, J., \& Hodges, J. R. (2011). Convergent grey and white matter evidence of C. (2007). The role of the ventrolateral frontal cortex in inhibitory oculomotor control. Brain, 130(6), 1525-37. orbitofrontal cortex changes related to disinhibition in behavioural variant frontotemporal dementia. Brain, 134(9), 2502-2512. doi:10.1093/brain/awr173

Iseki, C., Takahashi, Y., Wada, M., Kawanami, T., \& Kato, T. (2013). Subclinical Declines in the Verbal Fluency and Motor Regulation of Patients with AVIM (Asymptomatic Ventriculomegaly with Features of Idiopathic NPH on MRI): A Case-controlled Study. Internal Medicine, 52(15), 1687-1690. doi:10.2169/internalmedicine.52.8914

544 Luria, A. R. (1966). Higher cortical functions in man. Basic Books. 
Luria, A. R. (1980). Higher cortical Functions in man. Basic Books.

546 Martin, A. K., Barker, M. S., Gibson, E. C., \& Robinson, G. A. (2019). Response initiation and

Mikadze, Y. V. (2014). The principles of plasticity in Lurian neuropsychology. Psychology inhibition and the relationship with fluid intelligence across the adult lifespan. Archives

Mitsuhashi, S., Hirata, S., \& Okuzumi, H. (2018). Role of inner speech on the Luria hand test.

$$
\text { \&amp; Neuroscience, 7(4), 435-441. doi:10.3922/j.psns.2014.4.02 }
$$

$$
\text { Cogent Psychology, 5(1). doi:10.1080/23311908.2018.1449485 }
$$

Miziara, C. S., Manreza, M. L., Mansur, L., Reed, U. C., \& Buchpiguel, C. A. (2013). Sequential motor task (Lurias Fist-Edge-Palm Test) in children with benign focal epilepsy of childhood with centrotemporal spikes. Arquivos De Neuro-Psiquiatria, 71(6), 380-384.

doi:10.1590/0004-282x20130043

Mutha, P. K., Haaland, K. Y., \& Sainburg, R. L. (2012). The effects of brain lateralisation on motor control and adaptation. Journal of Motor Behavior, 44(6), 455-469.

Park, H., \& Moon, S. Y. (2014). Usefulness Of Fist-Edge-Palm Test In A Dementia Clinic.

563 Parris, B. A., Wadsley, M. G., Hasshim, N., Augustinova, M. \& Ferrand. L. (2019). An fMRI

564 study of response and semantic conflict in the Stroop task. Frontiers in Psychology, 10:

565 2426. doi: $10.3389 /$ fpsyg.2019.02426 
Perret, E. (1974). The left frontal lobe of man and the suppression of habitual responses in verbal categorical behaviour. Neuropsychologia, 12(3), 323-330. doi:10.1016/0028$3932(74) 90047-5$

Rao, H., Di, X., Chan, R. C., Ding, Y., Ye, B., \& Gao, D. (2008). A regulation role of the prefrontal cortex in the fist-edge-palm task: Evidence from functional connectivity analysis. NeuroImage, 41(4), 1345-1351. doi:10.1016/j.neuroimage.2008.04.026

Robbins, T. (2007). Shifting and stopping: Fronto-striatal substrates, neurochemical modulation and clinical implications. Philosophical Transactions of the Royal Society B: Biological Sciences, 362(1481), 917-932. doi:10.1098/rstb.2007.2097

Robinson, G. A., Cipolotti, L., Walker, D. G., Biggs, V., Bozzali, M., \& Shallice, T. (2015). Verbal suppression and strategy use: A role for the right lateral prefrontal cortex? Brain, 138(4), 1084-1096. doi:10.1093/brain/awv003

Roca, M., Parr, A., Thompson, R., Woolgar, A., Torralva, T., Antoun, N., . . Duncan, J. (2010). Executive function and fluid intelligence after frontal lobe lesions. Brain, 133(1), 234247. doi:10.1093/brain/awp269

Serrien, D. J., \& Brown, P. (2003). The integration of cortical and behavioural dynamics during initial learning of a motor task. European Journal of Neuroscience, 17(5), 1098-1104. doi:10.1046/j.1460-9568.2003.02534.x

Stroop, J. R. (1935). Studies of interference in serial verbal reactions. Journal of Experimental Psychology, 18(6), 643-662. doi:10.1037/h0054651

Stuss, D. T., Floden, D., Alexander, M. P., Levine, B., \& Katz, D. (2001). Stroop performance in focal lesion patients: Dissociation of processes and frontal lobe lesion location. Neuropsychologia, 39(8), 771-786. doi:10.1016/s0028-3932(01)00013-6 
Szczepanski, S. M., \& Knight, R. T. (2014). Insights into human behavior from lesions to the prefrontal cortex. Neuron, 83(5), 1002-1018.

591 Trenerry, M. R., Crosson, B., DeBoe, J., \& Leber, W. R. (1989). Stroop neuropsychological 592 screening test manual. Lutz, FL: Psychological Assessment Resources.

593 Trochim, W.M. and Donnelly, J.P. (2006). The Research Methods Knowledge Base. 3rd Edition, $594 \quad$ Atomic Dog, Cincinnati, OH.

595 Umetsu, A., Okuda, J., Fujii, T., Tsukiura, T., Nagasaka, T., Yanagawa, I., . . Suzuki, K.

596 (2002). Brain Activation during the Fist-Edge-Palm Test: A Functional MRI Study.

$597 \quad$ NeuroImage, 17(1), 385-392. doi:10.1006/nimg.2002.1218

598 Van Veen, V., \& Carter, C. S. (2005). Separating semantic conflict and response conflict in the 599 Stroop task: A functional MRI study. Neuroimage, 27(3), 497-504.

600 Weiner, M. F., Hynan, L. S., Rossetti, H., \& Falkowski, J. (2011). Lurias three-step test: What is 601 it and what does it tell us? International Psychogeriatrics, 23(10), 1602-1606.

602 doi:10.1017/s1041610211000767

603 Zaytseva, Y., Korsakova, N., Gurovich, I. Y., Heinz, A., \& Rapp, M. A. (2014). Luria revisited:

604 Complex motor phenomena in first episode schizophrenia and schizophrenia spectrum disorders. Psychiatry Research, 220(1-2), 145-151. doi:10.1016/j.psychres.2014.08.009

606

607 
Table 1. Participant information

\begin{tabular}{|c|c|c|c|c|}
\hline & & Mean & Standard & Group Size \\
\hline & & & Deviation & \\
\hline \multicolumn{2}{|c|}{ Age } & 21 & 3.3 & 72 (100\%) \\
\hline \multicolumn{2}{|c|}{ Education Level (Years) } & 15.2 & 1.1 & $72(100 \%)$ \\
\hline \multirow[t]{2}{*}{ Gender } & Male & - & - & $27(38 \%)$ \\
\hline & Female & - & - & 45 (62\%) \\
\hline \multirow[t]{2}{*}{ Handedness } & Right & - & - & 63 (89\%) \\
\hline & Left & - & - & $9(11 \%)$ \\
\hline
\end{tabular}

608

609 
Table 2. Performance data for the neuropsychological measures employed

\begin{tabular}{c|cc}
\hline & Mean & Standard Deviation \\
\hline Score & 114.5 & 11.64 \\
\hline Hayling Suppression & 15.19 & 13.98 \\
Reaction Time Score & & \\
\hline Hayling Suppression & 7.25 & 5.16 \\
Error Score & & \\
\hline Stroop Score & & \\
\hline
\end{tabular}


Table 3. Descriptive statistics of the FEP scores

\begin{tabular}{|c|c|c|c|}
\hline & & Mean & SD \\
\hline Subtle & Self- & 1.36 & 2.31 \\
\hline \multirow[t]{4}{*}{ Errors } & tempo & & \\
\hline & 60bpm & 0.63 & 1.01 \\
\hline & 120bpm & 2.09 & 1.86 \\
\hline & Total & 8.05 & 3.52 \\
\hline Crude & Self- & 1.59 & 1.18 \\
\hline \multirow[t]{4}{*}{ Errors } & tempo & & \\
\hline & $60 b p m$ & 1.67 & 1.25 \\
\hline & 120bpm & 2.66 & 2.14 \\
\hline & Total & 5.92 & 2.82 \\
\hline Subtle & Self- & 0.71 & 0.63 \\
\hline
\end{tabular}

627

628 
Table 4. Table summarizing the results of the Kendall correlation analysis

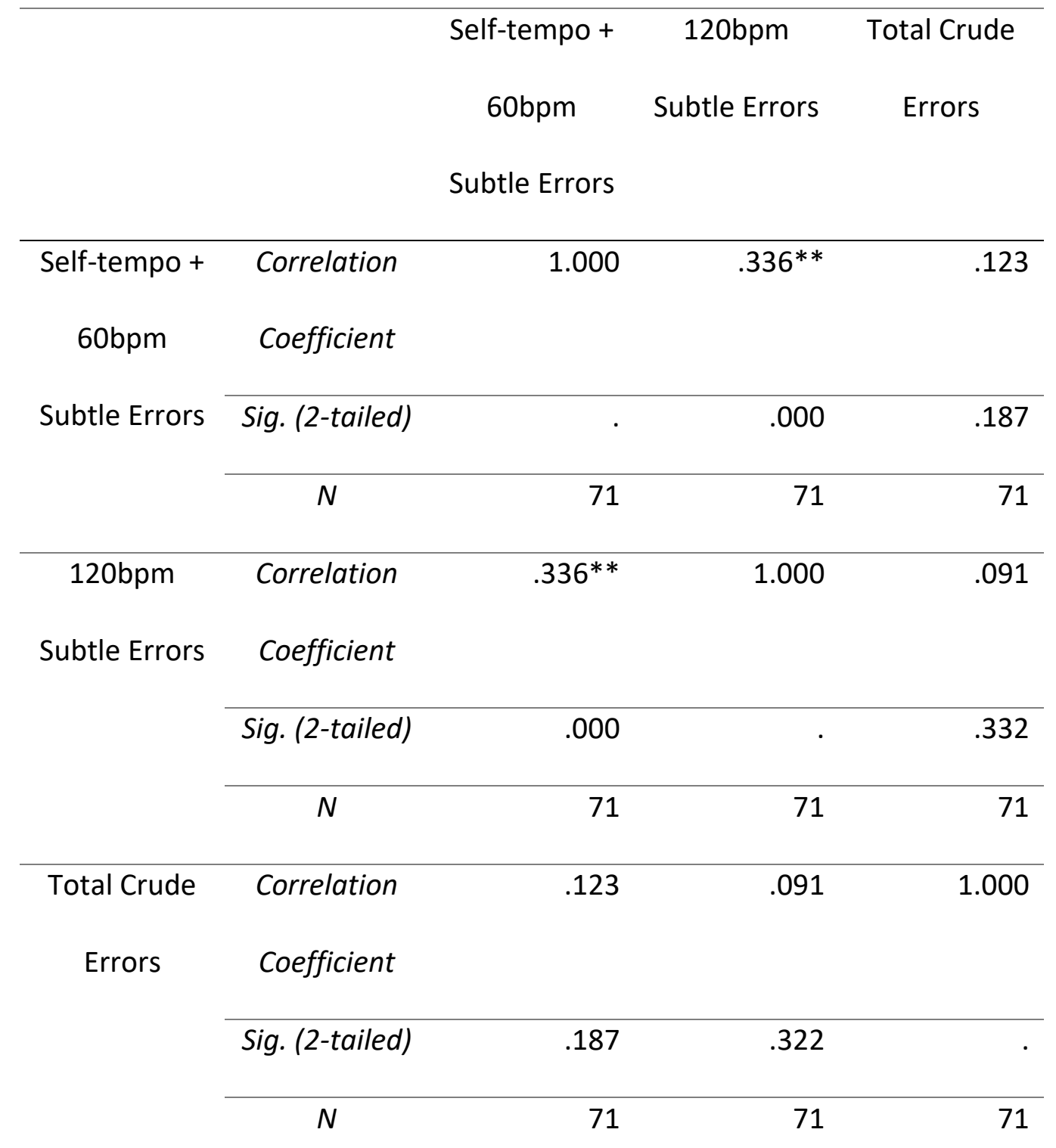

**. Correlation is significant at the 0.001 level (2-tailed). 
652 Figure Captions

653 Figure 1. Scatter plot depicting the multiple regression model for Overall performance on the 654 Fist-Edge-Palm test.

655 Figure 2. Scatter plot depicting the correlation between the overall FEP score and the Hayling 656 suppression error score.

657 Figure 3. Scatter plot depicting the multiple regression model for Total Crude Errors on the Fist658 Edge-Palm test.

659 Figure 4. Scatter plot depicting the correlation between the total crude error score and the 660 Hayling suppression error score.

661

662

663

664

665

666

667

668

669

670

671

672

673 


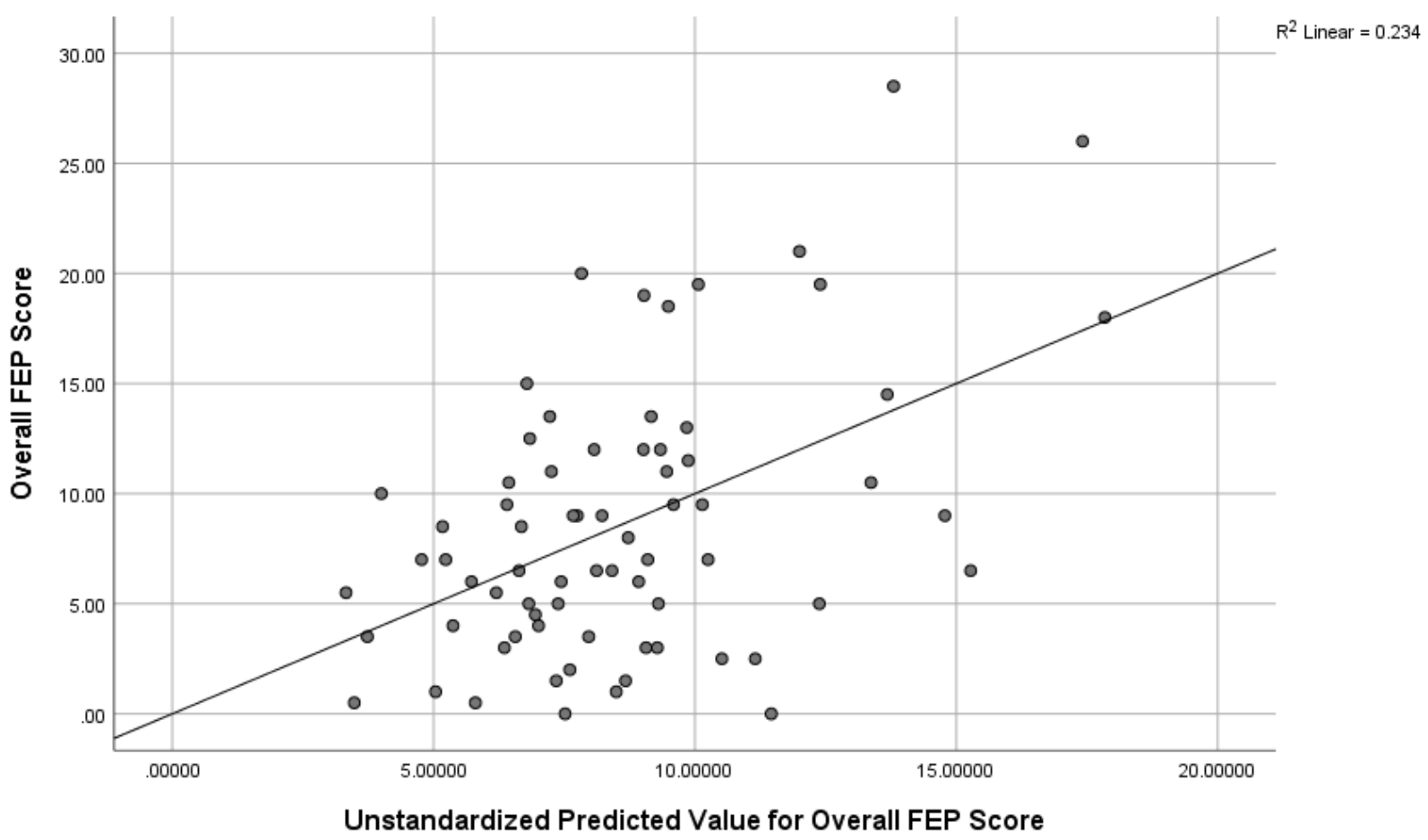

675

676

677

678

679

Figure 1. 


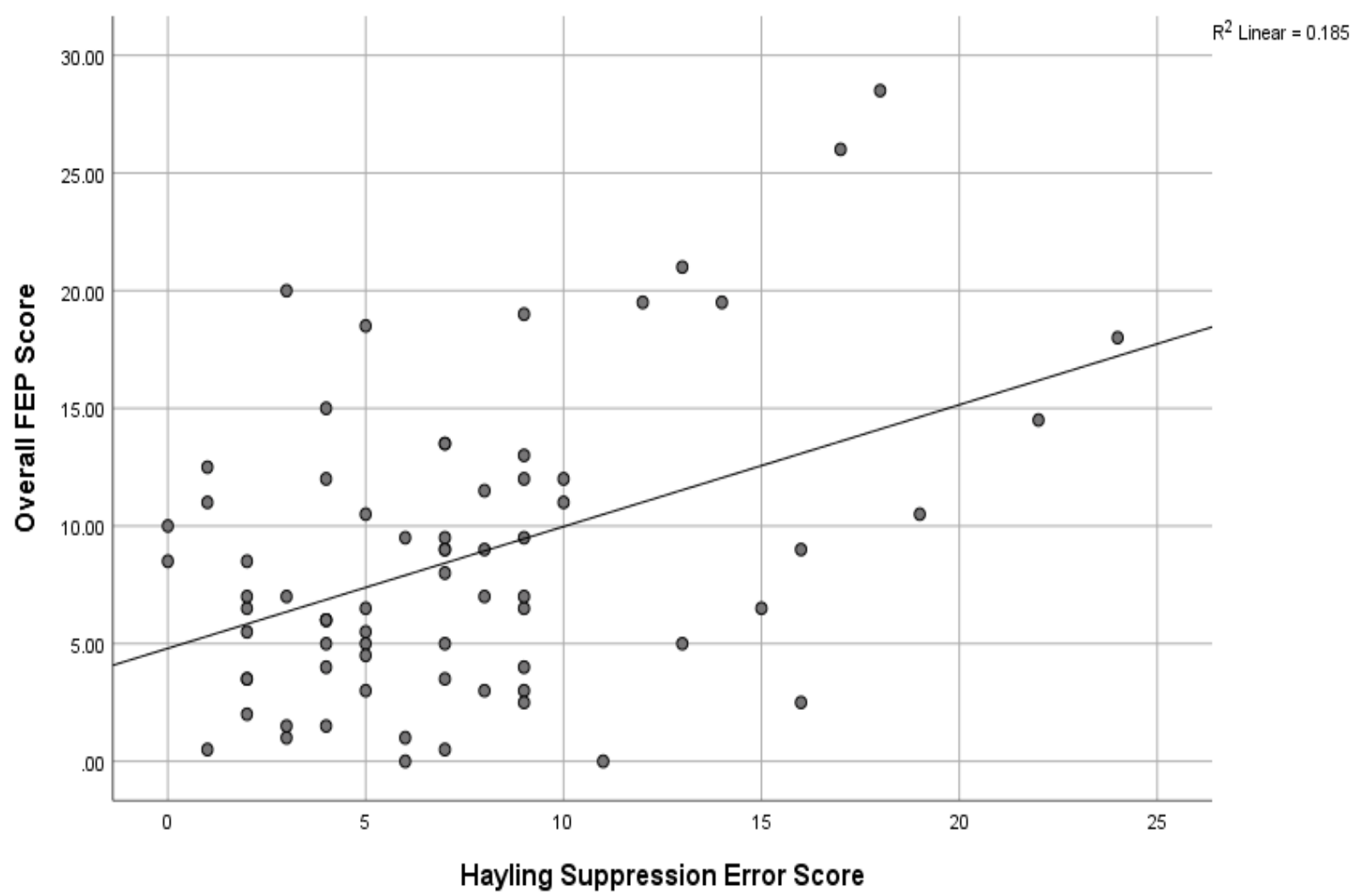

681

682 Figure 2.

683

684

685

686

687 
688

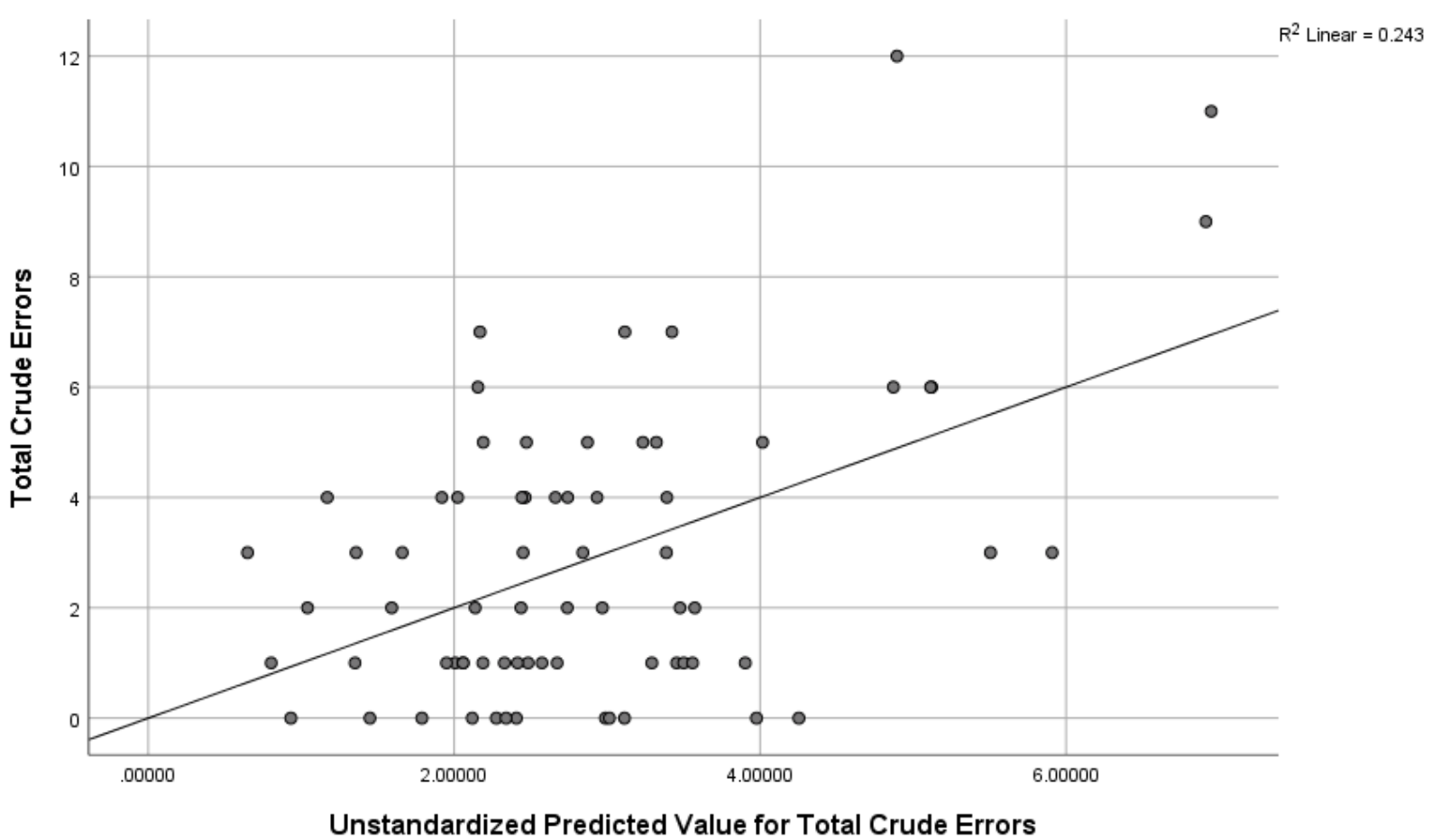

689

$690 \quad$ Figure 3.

691

692

693

694

695

696

697

698 


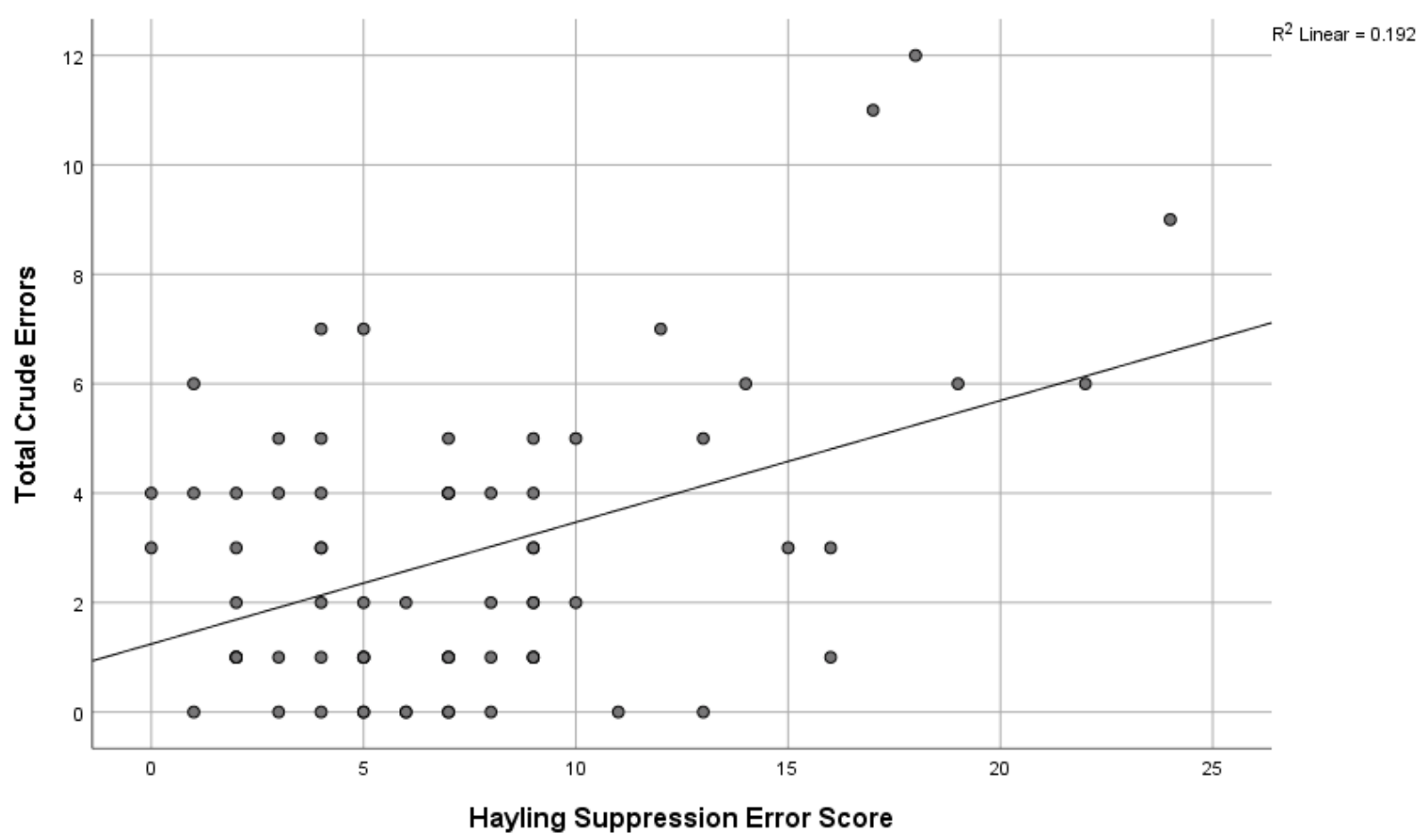

700

$701 \quad$ Figure 4. 